ISSN 1112-9867

Available online at http://www.jfas.info

\title{
NUMERICAL MODEL OF RAINWATER RUNOFF OVER THE CATCHMENT SURFACE AND TRANSPORT OF CONTAMINANT INCOMING TO WATER STREAM FROM SOIL
}

\author{
A. Chekalin*, M. Khramchenkov, V. Konyukhov, I. Konyukhov, A. Garaeva \\ Kazan Federal University
}

Published online: 08 August 2017

\begin{abstract}
This paper deals with development of computational schemes to solve of system equations with lagging argument governing the rainwater runoff along the surface catchment and transport of impurity which permeates into the water flow from soil at the certain areas of this surface. This system consists of two types of equations: the first of them describes the changes of water layer thickness over the slope surface given the precipitation and evaporation, and the second one - the mass transfer of impurity coming into the surface water during its filtration in zone of incomplete saturation of soil. Computational scheme to solve the system of nonlinear differential equations are developed with the finite-difference methods using a priori information about the solution behavior. The numerical model is implemented in the software for computer simulation of the processes with simultaneous graphic visualization of the computing results.
\end{abstract}

Keywords: Finite-Difference Schemes, Computational Algorithm, System Equations, Lagging Argument, Runoff, Transfer of Contaminant, Catchment

\section{INTRODUCTION}

In our previous publications [1] we have developed the mathematical model of runoff along the surface catchment taking account precipitation, evaporation, transport of impurity in

Author Correspondence, e-mail: Anatolii.Chekalin@kpfu.ru

doi: http://dx.doi.org/10.4314/jfas.v9i2s.64 
water, its filtration in zone of incomplete saturation of soil, sorbtion and adsorption of pollutant in soil. The total system of equations is subdivided on two sub-systems: the differential equations [2,3] governing the water runoff based on the concept of twodimensional kinematic wave $[4,5]$ and the equations describing the transport of contaminant incoming to the water stream from soil.

The present paper is a continuation of studies [1 - 3] and is devoted to develop the numerical and algorithmic models for calculation of the rainwater runoff. But before we begin the description of these results, we shall briefly formulate the mathematical model and the main notation here for completeness:

$$
\begin{aligned}
& \frac{\partial(U+\Theta)}{\partial t}+V_{x}+V_{y}=\varepsilon_{1}, \quad V_{x}=-U^{5 / 3} V_{1, x}, \quad V_{y}=-U^{5 / 3} V_{1, y}, \\
& \frac{\partial(C U)}{\partial t}+\frac{\partial\left(C V_{x}\right)}{\partial x}+\frac{\partial\left(C V_{y}\right)}{\partial y}=\beta-C \frac{\partial \Theta}{\partial t}, \quad h(1-m) \frac{\partial C_{s}}{\partial t}=h \beta_{s}-\beta, \\
& V_{1, x}=\frac{Z_{x}^{\prime}}{n \sqrt{|\nabla Z|},} V_{1, y}=\frac{Z_{y}^{\prime}}{n \sqrt{|\nabla Z|}}, \quad|\nabla Z|=\sqrt{Z_{x}^{\prime 2}+Z_{y}^{\prime 2}}, \quad \mathrm{Z}=\mathrm{H}+\mathrm{U}, \\
& \Theta=\sqrt{a^{2} t^{2}+b t}+a t, \quad a=0.25 m g \rho r^{2} / \mu, \quad b=0.5 m^{2} \sigma r \cos \alpha / \mu, \\
& \beta= \begin{cases}0, & \partial \Theta / \partial t=0, \\
\lambda\left(C_{s}-C\right), \quad U>0, \quad \beta_{s}=\left\{\begin{array}{ll}
0, & \\
\gamma\left(C-C_{s}\right), & \partial \Theta / \partial t>0,
\end{array} \quad, \quad(x, y) \notin D_{c},\right.\end{cases} \\
& U(x, y, 0)=0, \quad C(x, y, 0)=0,\left.\quad V_{n}\right|_{\Gamma_{w}}=0, \quad C_{s}(x, y, 0)=\left\{\begin{array}{l}
0 \\
C_{s}^{0}(x, y),
\end{array} \quad(x, y) \notin D_{c},\right.
\end{aligned}
$$

where $x$ and $y$ are spatial coordinates; $t$ is the time; $U(x, y, t)$ is the thickness of the water layer at the catchment surface; $\Theta(x, y, t)$ is water content in coil within zone of incomplete saturation («ZIS»); $m$ is the porosity of this area; $C(x, y, t)$ is the impurity concentration in water solution of the surface flow; $C_{s}(x, y, t)$ is the similar characteristic of the water solution in the soil thin boundary layer, by which water enters the soil from earth surface; $h$ is the thickness of boundary layer; $\mathbf{V}(x, y, t)$ is the velocity vector of the surface flow; $H(x, y)$ is the ground elevation above sea level; $n$ is the roughness coefficient of the catchment surface $D$ with boundary $\Gamma=\Gamma_{w}+\Gamma_{r}, \Gamma_{w}$ is the watershed line; $\Gamma_{r}$ is intersection of this line and the river surface at its lower course; $\left.V_{n}\right|_{\Gamma_{w}}$ is the normal velocity at the boundary $\Gamma_{w} ; D_{c}$ is the area of contamination source with the initial concentration $C_{s}^{0}(x, y) ; \varepsilon_{1}(x, y, t)$ is the function that specifies amount of precipitation and evaporation; $\rho$ is the water density; $g$ is 
the gravity acceleration; $r$ is the average radius of pores in zone of incomplete saturation; $\sigma$ is the surface tension coefficient; $\alpha$ is the wetting angle; $\mu$ is the viscosity of water solution; $\beta(x, y, t)$ is desorption of impurity from the surface of pores in soil by rainwater; $\beta_{s}(x, y, t)$ is the amount of the desorbed impurity in water, moving in the porous medium, per unit volume of soil; $\lambda$ and $\gamma$ are coefficients of desorption; $Z_{x}^{\prime}=\partial Z / \partial x ; Z_{y}^{\prime}=\partial Z / \partial y$.

So, the problem is formulated as follows: we need to determine the solution $U(x, y, t) \geq 0$, $\Theta(x, y, t), C(x, y, t)$ and $C_{s}(x, y, t)$ of equations (1) - (4) with initial and boundary conditions (5) and estimate the water flow rate in cross-section $\Gamma_{r}$ of river at its lower course.

\section{FINITE-DIFFERENCE METHODS AND COMPUTATIONAL ALGORITHM}

1. Finite-difference scheme. The system of nonlinear differential equations for the runoff along the surface catchment and transfer of impurity coming into the stream from soil is solved by the finite-difference methods proposed in [2]. The initial region solution is subdivided into the unit cells of the grid $D_{h}$ with the uniform step $h_{x}$ and $h_{y}$ along the axes $O x$ and $O y$ in such a way that the boundaries of cells were located at the boundary $\Gamma$. Let us designate boundary of grid and the time step as $\Gamma_{h}$ and $\tau$.

It is typical of the surface runoff that $|\nabla Z| \neq 0$, and, as a rule $|\nabla H|>>|\nabla U|$. Given the high uncertainty of the initial data let us assume in third and fourth equations (3) that $|\nabla h|=|\nabla u|$. It would seem that the derivatives $U_{x}^{\prime}, U_{y}^{\prime}$ can be ignored in these equations, and one assume that $Z_{x}^{\prime}=H_{x}^{\prime}, Z_{y}^{\prime}=H_{y}^{\prime}$. However, at certain conditions $U_{x}^{\prime}, U_{y}^{\prime}$ may have appreciable effects on the magnitude of the surface runoff. Therefore, the derivatives $Z_{x}^{\prime}, Z_{y}^{\prime}$ in third and fourth equalities (6) can be approximately written in the following form $Z_{x}^{\prime} \approx \sqrt{H_{x}^{\prime}+U_{x}^{\prime}} \sqrt{H_{x}^{\prime}}$, $Z_{y}^{\prime} \approx \sqrt{H_{y}^{\prime}+U_{y}^{\prime}} \sqrt{H_{y}^{\prime}}$. This allows to determine the values of these derivatives without error if the gradient $\nabla Z=\nabla H$ is directed along the corresponding coordinate axis, i.e. at $Z_{x}^{\prime}=0$ or $Z_{y}^{\prime}=0$. Taking account of the characteristic features of the considered problem, we develop the implicit difference scheme consisting of linearized algebraic equations in which flows of water and of solution across the boundaries of the unit cells are approximated by the following relationship: 


$$
\begin{aligned}
& \tilde{V}_{i \pm 1 / 2, j}= \begin{cases}U_{i \pm 1 / 2, j}^{t+\tau}\left(U_{i \pm 1 / 2, j}^{t}\right)^{2 / 3} V_{1, i \pm 1 / 2, j}, & \left(x_{i \pm 1 / 2}, y_{j}\right) \in D_{h} \\
0 \quad, & \left(x_{i \pm 1 / 2}, y_{j}\right) \in \Gamma_{h}\end{cases} \\
& \tilde{V}_{i, j \pm 1 / 2}= \begin{cases}U_{i, j \pm 1 / 2}^{t+\tau}\left(U_{i, j \pm 1 / 2}^{t}\right)^{2 / 3} V_{1, i, j \pm 1 / 2}, & \left(x_{i}, y_{j \pm 1 / 2}\right) \in D_{h}, \\
0 \quad, & \left(x_{i}, y_{j \pm 1 / 2}\right) \in \Gamma_{h},\end{cases} \\
& V_{c, i \pm 1 / 2, j}=C_{i \pm 1 / 2, j}^{t+\tau} \tilde{V}_{i \pm 1 / 2, j}^{t}, \quad V_{c, i, j \pm 1 / 2}=C_{i, j \pm 1 / 2}^{t+\tau} \tilde{V}_{i, j \pm 1 / 2}^{t}, \\
& V_{1, i \pm 1 / 2, j}=2 \frac{h_{y}}{h_{x}} \frac{\operatorname{sign}\left(Z_{i \pm 1, j}-Z_{i, j}\right) \sqrt{\left|Z_{i \pm 1, j}-Z_{i, j}\right|} \sqrt{\left|H_{i \pm 1, j}-H_{i, j}\right|}}{K_{i \pm 1 / 2, j}\left(n_{i, j}+n_{i+1, j}\right)} \\
& V_{1, i, j \pm 1 / 2}=2 \frac{h_{y}}{h_{x}} \frac{\operatorname{sign}\left(Z_{i, j \pm 1}-Z_{i, j}\right) \sqrt{\left|Z_{i, j \pm 1}-Z_{i, j}\right|} \sqrt{\left|H_{i, j \pm 1}-H_{i, j}\right|}}{K_{i, j \pm 1 / 2}\left(n_{i, j}+n_{i, j+1}\right)} \\
& K_{i \pm 1 / 2, j}=\left\{\begin{array}{ll}
\tilde{K}_{i \pm 1 / 2, j}, & \eta_{i \pm 1, j}<0, \\
\sqrt{\left(H_{i \pm 1, j}-H_{i, j}\right) / / h_{x}}, & \eta_{i \pm 1, j} \geq 0,
\end{array} \quad \eta_{i \pm 1, j}=\left(H_{i \pm 1, j}-H_{i \pm 1, j+1}\right)\left(H_{i \pm 1, j}-H_{i \pm 1, j-1}\right),\right. \\
& K_{i, j \pm 1 / 2}=\left\{\begin{array}{ll}
\tilde{K}_{i, j \pm 1 / 2}, & \eta_{i, j \pm 1}<0, \\
\sqrt{\left|\left(H_{i, j \pm 1}-H_{i, j}\right)\right| / h_{x}}, & \eta_{i, j \pm 1} \geq 0,
\end{array} \quad \eta_{i, j \pm 1}=\left(H_{i, j \pm 1}-H_{i-1, j \pm 1}\right)\left(H_{i, j \pm 1}-H_{i+1, j \pm 1}\right),\right. \\
& \tilde{K}_{i \pm 1 / 2, j}=\sqrt{2}\left\{\sqrt{\left(\frac{H_{i+1, j}-H_{i-1, j}}{2 h_{x}}\right)^{2}+\left(\frac{H_{i, j+1}-H_{i, j-1}}{2 h_{y}}\right)^{2}}+\sqrt{\left(\frac{H_{i+1 \pm 1, j}-H_{i-1 \pm 1, j}}{2 h_{x}}\right)^{2}+\left(\frac{H_{i \pm 1, j+1}-H_{i \pm 1, j-1}}{2 h_{y}}\right)^{2}}\right\}^{-1 / 2}, \\
& \tilde{K}_{i \pm 1 / 2, j}=\sqrt{2}\left\{\sqrt{\left(\frac{H_{i, j+1}-H_{i, j-1}}{2 h_{y}}\right)^{2}+\left(\frac{H_{i+1, j}-H_{i-1, j}}{2 h_{x}}\right)^{2}}+\sqrt{\left(\frac{H_{i, j \pm 1+1}-H_{i, j \pm 1-1}}{2 h_{y}}\right)^{2}+\left(\frac{H_{i+1, j \pm 1}-H_{i-1, j \pm 1}}{2 h_{x}}\right)^{2}}\right\}^{-1 / 2} .
\end{aligned}
$$

It must be kept in mind that the real profile of the surface catchment can include topographical data at $H_{i, j}<H_{i \pm 1, j}$ and $H_{i, j}<H_{i, j \pm 1}$, so the gradient of inclination is directed along the diagonal of the unit cell, and liquid flows from the cell $D_{i, j}$ into the one or even two cells adjoining to diagonal. In this case we need to determine not only the flows $\tilde{V}_{i \pm 1 / 2, j}$ and $\tilde{V}_{i, j \pm 1 / 2}$ but also the flows $\tilde{W}_{i \pm 1 / 2, j}$ from cell $D_{i, j}$ into cells $D_{i \pm 1, j \pm 1}$ and also $\tilde{W}_{i, j \pm 1 / 2}$ from cell $D_{i, j}$ into cells $D_{i \pm 1, j+1}$. To define these flows we introduce a new type of cells instead of the rectangular cells. Let $H_{i, j}>H_{i+1, j+1}$. If both inequalities $H_{i+1, j+1}<H_{i+1, j}$ and $H_{i+1, j+1}<H_{i, j+1}$ 
are satisfied then the main fluid flow is directed along the diagonal from cell $D_{i, j}$ to the cell $D_{i+1, j+1}$, and it is necessary to define the values of $\tilde{W}_{i+1 / 2, j}$ and $\tilde{W}_{c, i+1 / 2, j}$. It should be noted that if any one or more of these three conditions is not met, then $\tilde{W}_{i+1 / 2, j}=0$ and $\tilde{W}_{c, i+1 / 2, j}=0$. The modified cells $D_{i, j}, D_{i+1, j}, D_{i, j+1}$ and $D_{i+1, j+1}$ are shown in the Fig. 1 .

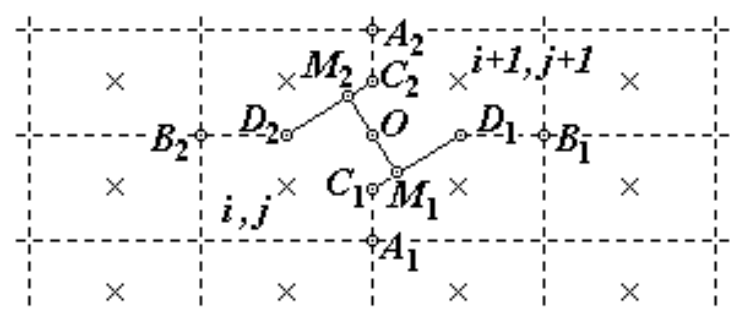

Fig.1. Modification of cells in the case when the runoff is directed along the diagonal from the cell $D_{i, j}$ to the cell $D_{i+1, j}$

By the construction of these cells, the boundary $M_{1} M_{2}$ is orthogonal with the segments $C_{1} D_{1}$ and $C_{2} D_{2}$ which connect the midpoint of the respective sides of the rectangular cells. The length $l$ of the segment $M_{1} M_{2}$ is equal to $l=h_{x} h_{y} / \sqrt{h_{x}^{2}+h_{y}^{2}}$, and the «diagonal» flows are determined by formulas:

$$
\begin{aligned}
& \tilde{W}_{i+1 / 2, j}=U_{i, j}^{t+\tau}\left(U_{i, j}^{t}\right)^{2 / 3} \omega_{i+1 / 2, j}, \quad \tilde{W}_{c, i+1 / 2, j}=\tilde{W}_{i+1 / 2, j} C_{i, j}^{t+\tau}, \\
& \omega_{i+1 / 2, j}=-2 l \sqrt{\left|\left(Z_{i+1, j+1}-Z_{i, j}\right)\right|}\left(n_{i, j}+n_{i+1, j+1}\right)^{-1}\left(h_{x}^{2}+h_{y}^{2}\right)^{-1 / 4} .
\end{aligned}
$$

Let us define the flows $v_{1}, v_{2}, v_{3}$ and $v_{4}$ through the boundaries $A_{1} C_{1} M_{1}, M_{1} D_{1} B_{1}, A_{2} C_{2} M_{2}$ and $M_{2} D_{2} B_{2}$, respectively. The components of these flows are defined from the relationships (6), (8) in segments $A_{1} C_{1}, D_{1} B_{1}, A_{2} C_{2}$ and $D_{2} B_{2}$, but in doing so, their magnitudes must become less by half due to decreasing of length of boundaries. Consider now the other segments $C_{1} M_{1}, M_{1} D_{1}, C_{2} M_{2}$ and $M_{2} D_{2}$. For $H_{i, j} \geq H_{i+1, j}$ the flow is directed from the cell $D_{i, j}$ into the cell $D_{i+1, j+1}$ through the cell $D_{i+1, j}$. We can assume that boundary $C_{1} D_{1}$ goes along the stream-line and the normal flow velocity at this boundary is zero. Hence, the flows across the boundaries of $A_{1} C_{1} M_{1}$ and $M_{1} D_{1} B_{1}$ is equal to $0.5 \tilde{V}_{i \pm 1 / 2, j}$ and $0.5 \tilde{V}_{i, j \pm 1 / 2}$, respectively. If $H_{i, j}<H_{i+1, j}$ then the normal and tangent components $\theta_{1}$ and $\phi_{1}$ of the gradient surface inclination at $C_{1} D_{1}$ can be approximated in the following form: 


$$
\theta_{1}=\left[\frac{h_{y}}{h_{x}}\left(H_{i+1, j}-H_{i, j}\right)+\frac{h_{x}}{h_{y}}\left(H_{i+1, j}-H_{i+1, j+1}\right)\right]\left(h_{x}^{2}+h_{y}^{2}\right)^{-1 / 2}, \quad \phi_{1}=\left[H_{i+1, j+1}-H_{i, j}\right]\left(h_{x}^{2}+h_{y}^{2}\right)^{-1 / 2} .
$$

Similarly, the approximations for the normal and tangent components $\theta_{2}, \phi_{2}$ of this gradient at the boundary $C_{2} D_{2}$ for $H_{i, j}<H_{i, j+1}$ are:

$\theta_{2}=\left[\frac{h_{x}}{h_{y}}\left(H_{i, j+1}-H_{i, j}\right)+\frac{h_{y}}{h_{x}}\left(H_{i, j+1}-H_{i+1, j+1}\right)\right]\left(h_{x}^{2}+h_{y}^{2}\right)^{-1 / 2}, \phi_{2}=\phi_{1}$.

Since the segments $C_{1} M_{1}, \quad C_{2} M_{2}$ have the same length $l_{1}=0.5 h_{x}^{2} \sqrt{h_{x}^{2}+h_{y}^{2}}$, and $M_{1} D_{1}=M_{2} C_{2}=l_{2}=0.5 h_{y}^{2} \sqrt{h_{x}^{2}+h_{y}^{2}}$, the flow $v_{1}, v_{2}, v_{3}, v_{4}$ across the boundaries $A_{1} C_{1} M_{1}$, $M_{1} D_{1} B_{1}, A_{2} C_{2} M_{2}, M_{2} D_{2} B_{2}$ are determined by the relationships:

$v_{1}=U_{i+1, j}^{t+\tau}\left(U_{i+1, j}^{t}\right)^{2 / 3}\left(0.5 V_{1, i+1 / 2, j}+l_{1}\left(\theta_{1}^{2}+\phi_{1}^{2}\right)^{-1 / 4}\right)$,

$v_{2}=U_{i+1, j}^{t+\tau}\left(U_{i+1, j}^{t}\right)^{2 / 3}\left(0.5 V_{1, i+1, j+1 / 2}+l_{2}\left(\theta_{1}^{2}+\phi_{1}^{2}\right)^{-1 / 4}\right)$,

$v_{3}=U_{i, j+1}^{t+\tau}\left(U_{i, j+1}^{t}\right)^{2 / 3}\left(0.5 V_{1, i, j+1 / 2}+l_{1}\left(\theta_{2}^{2}+\phi_{2}^{2}\right)^{-1 / 4}\right)$,

$v_{4}=U_{i, j+1}^{t+\tau}\left(U_{i, j+1}^{t}\right)^{2 / 3}\left(0.5 V_{1+1 / 2, i, j+1}+l_{2}\left(\theta_{2}^{2}+\phi_{2}^{2}\right)^{-1 / 4}\right)$.

Thus, instead of the formulas (6) we have:

$$
\begin{aligned}
& \tilde{V}_{i+1 / 2, j}=\left\{\begin{array}{l}
v_{1},\left(x_{i+1 / 2}, y_{j}\right) \in D_{h}, \\
0,\left(x_{i+1 / 2}, y_{j}\right) \in \Gamma_{h},
\end{array} \quad \tilde{V}_{i+1, j+1 / 2}=\left\{\begin{array}{l}
v_{2},\left(x_{i+1}, y_{j+1 / 2}\right) \in D_{h}, \\
0,\left(x_{i+1}, y_{j+1 / 2}\right) \in \Gamma_{h},
\end{array}\right.\right. \\
& \tilde{V}_{i, j+1 / 2}=\left\{\begin{array}{l}
v_{3},\left(x_{i}, y_{j+1 / 2}\right) \in D_{h}, \\
0,\left(x_{i}, y_{j+1 / 2}\right) \in \Gamma_{h},
\end{array} \quad \tilde{V}_{i+1 / 2, j+1}= \begin{cases}v_{4},\left(x_{i+1 / 2}, y_{j+1}\right) \in D_{h}, \\
0,\left(x_{i+1 / 2}, y_{j+1}\right) \in \Gamma_{h} .\end{cases} \right.
\end{aligned}
$$

It is obvious that area of four modified unit cells are changed, namely, $S_{i, j}=S_{i+1, j+1}=9 / 8 h_{x} h_{y}, S_{i+1, j}=S_{i, j+1}=7 / 8 h_{x} h_{y}$. Note that if all the diagonal flows are equal to zero in the cell $S_{i, j}$, then this cell doesn't change and its area is $S_{i, j}=h_{x} h_{y}$. The values of other the «diagonal» flows $\tilde{W}_{i-1 / 2, j}$ and $\tilde{W}_{i, j \pm 1 / 2}$ are determined in a similar way. It should be noted also that, given the great uncertainty of the input data, we can use more simple approximation for the flows across these boundaries, neglecting the second term in the brackets in (10), i.e. ignoring the flows across the boundaries $C_{1} M_{1} D_{1}$ and $C_{2} M_{2} D_{2}$. 
As is known, the derivatives in the convective terms of the transfer equations should be approximated by the upstream finite differences, using the values of the unknown function at the unit cell from which the liquid flows out:

$$
\begin{aligned}
& \left(U_{i+1 / 2, j}^{t+\tau}\left(U_{i+1 / 2, j}^{t}\right)^{2 / 3}=\left\{\begin{array}{ll}
U_{i, j}^{t+\tau}\left(U_{i, j}^{t}\right)^{2 / 3}, & V_{1, i+1 / 2, j} \leq 0, \\
U_{i+1, j}^{t+\tau}\left(U_{i+1, j}^{t}\right)^{2 / 3}, & V_{1, i+1 / 2, j}>0,
\end{array} \quad C_{i+1 / 2, j}^{t+\tau}= \begin{cases}C_{i, j}^{t+\tau}, & V_{1, i+1 / 2, j} \leq 0, \\
C_{i+1, j}^{t+\tau}, & V_{1, i+1 / 2, j}>0,\end{cases} \right.\right. \\
& U_{i-1 / 2, j}^{t+\tau}\left(U_{i-1 / 2, j}^{t}\right)^{2 / 3}=\left\{\begin{array}{ll}
U_{i-1, j}^{t+\tau}\left(U_{i-1, j}^{t}\right)^{2 / 3}, & V_{1, i-1 / 2, j} \geq 0, \\
U_{i, j}^{t+\tau}\left(U_{i, j}^{t}\right)^{2 / 3}, & V_{1, i-1 / 2, j}<0,
\end{array} C_{i-1 / 2, j}^{t+\tau}= \begin{cases}C_{i-1, j}^{t+\tau}, & V_{1, i-1 / 2, j} \geq 0, \\
C_{i, j}^{t+\tau}, & V_{1, i-1 / 2, j}<0,\end{cases} \right. \\
& U_{i, j+1 / 2}^{t+\tau}\left(U_{i, j+1 / 2}^{t}\right)^{2 / 3}=\left\{\begin{array}{ll}
U_{i, j}^{t+\tau}\left(U_{i, j}^{t}\right)^{2 / 3}, & V_{1, i, j+1 / 2} \leq 0, \\
U_{i, j+1}^{t+\tau}\left(U_{i, j+1}^{t}\right)^{2 / 3}, & V_{1, i, j+1 / 2}>0,
\end{array} \quad C_{i, j+1 / 2}^{t+\tau}= \begin{cases}C_{i, j}^{t+\tau}, & V_{1, i, j+1 / 2} \leq 0, \\
C_{i, j+1}^{t+\tau}, & V_{1, i, j+1 / 2}>0,\end{cases} \right. \\
& U_{i, j-1 / 2}^{t+\tau}\left(U_{i, j-1 / 2}^{t}\right)^{2 / 3}= \begin{cases}U_{i, j-1}^{t+\tau}\left(U_{i, j-1}^{t}\right)^{2 / 3}, & V_{1, i, j-1 / 2}<0, \\
U_{i, j}^{t+\tau}\left(U_{i, j}^{t}\right)^{2 / 3}, & V_{1, i, j-1 / 2} \geq 0,\end{cases}
\end{aligned}
$$

The conservative finite-difference scheme, approximating differential equations (1), (2) in the grid-points of the discrete domain $D_{h}$ can be written as:

$$
\begin{aligned}
& \tau \Lambda[\tilde{V}+\tilde{W}]_{i, j}=S_{i, j}\left(U_{i, j}^{t+\tau}-U_{i, j}+\Theta_{i, j}^{t+\tau}-\Theta_{i, j}-\tau \varepsilon_{1, i, j}\right), \\
& \varepsilon_{1, i, j}=\frac{1}{\tau} \int_{t}^{t+\tau} \varepsilon_{1} d \tau, \quad \tau \Lambda\left[V_{c}+W_{c}\right]_{i, j}=S_{i, j}\left(C_{i, j}^{t+\tau} U_{i, j}^{t+\tau}-C_{i, j}^{t} U_{i, j}^{t}+\left(\Theta_{i, j}^{t+\tau}-\Theta_{i, j}^{t}\right) C_{i, j}^{t+\tau}-\tau \beta_{i, j}^{t+\tau}\right), \\
& h(1-m)\left(C_{s, i, j}^{t+\tau}-C_{s, i, j}^{t}\right)=\tau\left(h \beta_{s, i, j}^{t+\tau}-\beta_{i, j}^{t+\tau}\right),
\end{aligned}
$$

where $\Lambda[\tilde{Y}]_{i, j}=\tilde{Y}_{i+1 / 2, j}+\tilde{Y}_{i-1 / 2, j}+\tilde{Y}_{i, j+1 / 2}+\tilde{Y}_{i, j-1 / 2}$. It is convenient to rewrite the equations (13) and (14) in the following form:

$$
\begin{aligned}
& C_{s, i, j}^{t+\tau}=C_{i, j}^{t+\tau}+b_{c}\left(C_{s, i, j}^{t}-C_{i, j}^{t+\tau}\right), \quad b_{c}=\frac{\tau \gamma}{1-m+\tau \gamma}, \\
& C_{i, j}^{t+\tau}\left(U_{i, j}^{t+\tau}+\Theta_{i, j}^{t+\tau}-\Theta_{i, j}^{t}+\tau a_{c} \lambda\right)-C_{i, j}^{t} U_{i, j}^{t}-\tau a_{c} \lambda C_{s, i, j}^{t}=\tau \Lambda\left[V_{c}+W_{c}\right]_{i, j} / S_{i, j}, \\
& a_{c}=\frac{1-m}{1-m+\tau(\gamma+\lambda / \lambda h h)} .
\end{aligned}
$$

As it is shown in our paper [1], in the initial time the surface layer water is not formed as precipitation is completely absorbed into the zone of incomplete saturation and in this case the lag time $t_{0}$ at the arising of surface runoff water and the retarded argument $\tau_{d}$ are determined from equations: 
$t_{1}=\frac{b}{2 a^{2}}\left(\frac{\varepsilon_{1}\left(t_{0}\right)-a}{\sqrt{\varepsilon_{1}^{2}\left(t_{0}\right)-2 a \varepsilon_{1}\left(t_{0}\right)}}-1\right), \quad A\left(t_{0}\right)=\frac{b}{2 a}\left(-1+\frac{\varepsilon_{1}\left(t_{0}\right)}{\sqrt{\varepsilon_{1}^{2}\left(t_{0}\right)-2 a \varepsilon_{1}\left(t_{0}\right)}}\right)$

in each cell. Here $t_{1}=t_{0}-\tau_{d}$. It should be noted that the intensity $\varepsilon_{1}$ of precipitation and evaporation of water is usually determined by its approximate average value over the area and time. Therefore, the lag time $t_{0}$ and the retarded argument $\tau_{d, i, j}$ can be obtained at $\varepsilon_{1}>2 a$ from equalities [1]:

$t_{0}=\frac{b}{2 a \varepsilon_{1}}\left(-1+\frac{\varepsilon_{1}}{\sqrt{\varepsilon_{1}^{2}-2 a \varepsilon_{1}}}\right), \quad \tau_{d}=t_{0}-t_{1}=\frac{b}{2 a^{2}}\left(1-\frac{a}{\varepsilon_{1}}-\sqrt{1-\frac{2 a}{\varepsilon_{1}}}\right)$.

If parameters $a, b$ and $\varepsilon_{1}$ in Eq. (17) are functions of the coordinates $(x, y)$ then the values of $t_{0}$ and $\tau_{d}$ depend on the mesh node $\left(x_{i}, y_{j}\right)$. In this case a minimum value $\overline{t_{0}}=\min _{D_{h}} t_{0}\left(x_{i}, y_{j}\right)$ is defined, and from this point on it is assumed by the new initial moment in computation. Obviously, $\overline{t_{0}}=0$ if $a=b=0$ in some mesh nodes (e.g., if they located in the river bed). In those unit cells where the lag time is greater than $\overline{t_{0}}$, it is required to recalculate the values of $\overline{t_{0}}$ with account for possible inflows from the surrounding cells. Actually, in computation with the use of the implicit scheme already at the first point of time $\overline{t_{0}}+\tau$, the flows of water arise across the boundaries of neighboring grid cells in which $t_{0}=\bar{t}_{0}$. Let, for example, $t_{0}>\overline{t_{0}}$ in the unit cell $D_{i, j}$ and water enters into it from the surrounding cells. Hereinafter, we denote the total amount of all flows coming into the grid cell as $w^{+}$, and the intensity of water inflow into the cell at $t_{0}>\bar{t}_{0}$ as $\varepsilon_{w}=w^{+}+\varepsilon_{1, i, j}$. Since at $t_{0}<\overline{t_{0}}$ water, inflowing into the unit cell $D_{i, j}$, is completely absorbed by the soil of ZIS, so the water amount $A\left(t_{0}\right)$ in this zone at time $t_{0}$ is defined as: $A\left(t_{0}\right)=A\left(\overline{t_{0}}\right)+\left(t_{0}-\overline{t_{0}}\right) \varepsilon_{w}$, where $A\left(\overline{t_{0}}\right)$ is amount of water in ZIS at $t=\overline{t_{0}}$. In this case $A\left(\overline{t_{0}}\right)=\int_{0}^{\bar{t}_{0}} \varepsilon_{1, i, j} d t$. Thus, the new values $t_{0}$ and $\tau_{d}$ are determined from the same system equations (8) and (9), but with other right-hand parts. The solution of this system is:

$t_{0}=\overline{t_{0}}+\frac{b}{2 a \varepsilon_{w}}\left(\frac{1}{\sqrt{1-2 a / \varepsilon_{w}}}-1\right)-\frac{A\left(\overline{t_{0}}\right)}{\varepsilon_{w}}$.

If $t_{0}-\bar{t}_{0}<\tau$ then the retarded argument $\tau_{d, i, j}$ is calculated from formula 


$$
\tau_{d, i, j}=\overline{t_{0}}+\frac{b}{2 a^{2}}\left(1-a / \varepsilon_{w}-\sqrt{1-2 a / \varepsilon_{w}}\right)-\frac{A\left(\overline{t_{0}}\right)}{\varepsilon_{w}}
$$

and the water content $\Theta_{i, j}^{t+\tau}$ in zone of incomplete saturation is defined by equality [1]

$$
\Theta=\sqrt{a^{2}\left(t-\tau_{d}\right)^{2}+b\left(t-\tau_{d}\right)}+a\left(t-\tau_{d}\right)
$$

at $\tau_{d}=\tau_{d, i, j}$. The thickness $U_{i, j}^{t+\tau}$ of water layer is calculated from the algebraic equation (12) for discrete time $\bar{\tau}=\tau-\left(t_{0}-\overline{t_{0}}\right)$.

If $t_{0}>\overline{t_{0}}+\tau$ then the above procedure computation of values $t_{0}$ and $\tau_{d, i, j}$ is similarly repeated in the next time point.

In much the same way as reported previously the problem is solved at formation of such regions on the surface catchment in which the water layer temporarily disappears in result of its absorbtion by soil. Let $\Theta_{i, j}^{t+\tau}$ be the water amount in ZIS, calculated from formula (20). Then from the inequality

$$
\tau w^{+} / S_{i, j}+U_{i, j}-\Theta_{i, j}^{t+\tau}+\Theta_{i, j}+\tau \varepsilon_{1, i, j}<0
$$

it follows that at $U_{i, j}>0$ the rainwater is completely adsorbed and $U_{i, j}=0$. Therefore, $\Theta_{i, j}^{t+\tau}$ should be calculated not from formula (20) but from equation

$$
\Theta_{i, j}^{t+\tau}=U_{i, j}+\Theta_{i, j}+\tau \varepsilon_{1, i, j} \tau+w^{+} / S_{i, j} .
$$

If $U_{i, j}=0$ then the lag time $t_{0}$ is defined from formula (18) at $A\left(\overline{t_{0}}\right)=\Theta_{i, j}$ and, if it is necessary the values $\tau_{d, i, j}$ are also recomputed from relationship (19). As well as we do earlier, at $t_{0}-\overline{t_{0}}<\tau$ the retarded argument $\tau_{d, i, j}$ is computed from formula (19), the amount of water $\Theta_{i, j}^{t+\tau}$ in ZIS - in accordance with equality (20) for the current value of $\tau_{d, i, j}$, and the thickness of the water layer $U_{i, j}^{t+\tau}$ - from the difference equations (12) at the time point $\bar{\tau}=\tau-\left(t_{0}-\bar{t}_{0}\right)$. At $\left.t_{0}>\overline{t_{0}}\right)+\tau$ the thickness $U_{i, j}^{t+\tau}=0$, the mesh function $\Theta_{i, j}^{t+\tau}$ is defined from formula (21), and the values of $t_{0}$ and $\tau_{d, i, j}$ are calculated all over again at the next point in time.

With the appearance of region $D_{c r}$ with vanishing water layer the concentration of contaminant becomes equal to zero: $C_{i, j}^{t+\tau}=0$. However, the impurity can flow into the grid cells located at the boundary $\Gamma_{c r}$ of this region from the adjacent unit cells. This contaminant is completely absorbed by soil of ZIS. Let us denote the total quantity of all the incoming 
flows with the impurity into the cell $D_{i, j}$ as $w_{c}^{+}$, and the impurity concentration of in the water solution which enters into the zone of incomplete saturation over time $\tau$ as $\bar{C}$. Now we can write the following relationship for $\bar{C}: \bar{C}=\left(C_{i, j} U_{i, j}+\tau w_{c}^{+} / S_{i, j}\right) /\left(\Theta_{i, j}^{t+\tau}-\Theta_{i, j}\right)$, where $\left(\Theta_{i, j}^{t+\tau}-\Theta_{i, j}\right)$ is calculated from equation (21). Then the function $C_{s, i, j}^{t+\tau}$ is defined as:

$C_{s, i, j}^{t+\tau}=C_{s, i, j}^{t}-\frac{\tau \gamma}{1-m+\tau \gamma}\left(C_{s, i, j}^{t}-\bar{C}\right)$

2. Computational algorithm. Difference scheme (6) - (22) consists of two systems of linear algebraic equations. The first of them is obtained in result of linearization of the implicit difference scheme in respect to the functions $U$ and does not depend on concentration $C$. The second one is used to determine of $C$ from system of linear equations after computation of $U$. Both systems can be transformed to diagonal form by renumbering the grid cells in a different way: either in descending order of $Z$ or of $H$. This allows us to solve equations sequentially starting with the first equation corresponding to the maximum height of the catchment relief.

The first procedure requires the transformation of the system matrix at every time point. The second approach allows us to do such transformation only once in the beginning of the problem solution, that significantly reduces the calculations required and overall computation time. However, in those situations when $\left(H_{i+1, j}-H_{i, j}\right)\left(Z_{i+1, j}-Z_{i, j}\right)<0$, the runoff can goes upslope (e.g. at flooding of the river banks during the period of overflow), so that the coefficient matrix of a system of linear algebraic equations loses its diagonal form. To avoid this, we impose an additional conditions on $U_{i \pm 1 / 2, j}^{t+\tau} \cdot\left(U_{i \pm 1 / 2, j}^{t}\right)^{2 / 3}, U_{i, j \pm 1 / 2}^{t+\tau} \cdot\left(U_{i, j \pm 1 / 2}^{t}\right)^{2 / 3}, C_{i \pm 1 / 2, j}^{t+\tau}$, $C_{i, j \pm 1 / 2}^{t+\tau}$ in the relationships (11):

$$
\begin{gathered}
U_{i+1 / 2, j}^{t+\tau} \cdot\left(U_{i+1 / 2, j}^{t}\right)^{2 / 3}=\left\{\begin{array}{l}
U_{i, j}^{t+\tau} \cdot\left(U_{i, j}^{t}\right)^{2 / 3}, V_{1, i+1 / 2, j} \leq 0, H_{i, j}>H_{i+1, j}, \\
U_{i+1, j}^{t+\tau} \cdot\left(U_{i+1, j}^{t}\right)^{2 / 3}, V_{1, i+1 / 2, j}>0, H_{i, j}<H_{i+1, j}, \\
U_{i, j}^{t} \cdot\left(U_{i, j}^{t}\right)^{2 / 3}, V_{1, i+1 / 2, j} \leq 0, H_{i, j}<H_{i+1, j}, \\
U_{i+1, j}^{t} \cdot\left(U_{i+1, j}^{t}\right)^{2 / 3}, V_{1, i+1 / 2, j}>0, H_{i, j}>H_{i+1, j},
\end{array}\right. \\
U_{i-1 / 2, j}^{t+\tau} \cdot\left(U_{i-1 / 2, j}^{t}\right)^{2 / 3}= \begin{cases}U_{i-1, j}^{t+\tau} \cdot\left(U_{i-1, j}^{t}\right)^{2 / 3}, V_{1, i-1 / 2, j} \leq 0, H_{i, j}>H_{i+1, j}, \\
U_{i, j}^{t+\tau} \cdot\left(U_{i, j}^{t}\right)^{2 / 3}, & V_{1, i+1 / 2, j}>0, H_{i, j}<H_{i+1, j}, \\
U_{i-1, j}^{t} \cdot\left(U_{i-1, j}^{t}\right)^{2 / 3}, & V_{1, i-1 / 2, j} \leq 0, H_{i, j}<H_{i+1, j}, \\
U_{i, j}^{t} \cdot\left(U_{i, j}^{t}\right)^{2 / 3}, & V_{1, i+1 / 2, j}>0, H_{i, j}<H_{i+1, j},\end{cases}
\end{gathered}
$$




$$
\begin{aligned}
& U_{i, j+1 / 2}^{t+\tau} \cdot\left(U_{i, j+1 / 2}^{t}\right)^{2 / 3}=\left\{\begin{array}{ll}
U_{i, j}^{t+\tau} \cdot\left(U_{i, j}^{t}\right)^{2 / 3}, & V_{1, i, j+1 / 2} \leq 0, H_{i, j}>H_{i, j+1}, \\
U_{i, j+1}^{t+\tau} \cdot\left(U_{i, j+1}^{t}\right)^{2 / 3}, & V_{1, i, j+1 / 2}>0, H_{i, j}<H_{i, j+1}, \\
U_{i, j}^{t} \cdot\left(U_{i, j}^{t}\right)^{2 / 3}, & V_{1, i, j+1 / 2} \leq 0, H_{i, j}<H_{i, j+1}, \\
U_{i, j+1}^{t} \cdot\left(U_{i, j+1}^{t}\right)^{2 / 3}, & V_{1, i, j+1 / 2}>0, H_{i, j}>H_{i, j+1},
\end{array},\right. \\
& U_{i, j-1 / 2}^{t+\tau} \cdot\left(U_{i, j-1 / 2}^{t}\right)^{2 / 3}= \begin{cases}U_{i, j-1}^{t+\tau} \cdot\left(U_{i, j-1}^{t}\right)^{2 / 3}, V_{1, i, j-1 / 2}<0, H_{i, j}<H_{i, j-1}, \\
U_{i, j}^{t+\tau} \cdot\left(U_{i, j}^{t}\right)^{2 / 3}, V_{1, i, j-1 / 2} \geq 0, H_{i, j}>H_{i, j-1}, \\
U_{i, j-1}^{t} \cdot\left(U_{i, j-1}^{t}\right)^{2 / 3}, V_{1, i, j-1 / 2}<0, H_{i, j}>H_{i, j-1}, \\
U_{i, j}^{t} \cdot\left(U_{i, j}^{t}\right)^{2 / 3}, V_{1, i, j-1 / 2} \geq 0, H_{i, j}<H_{i, j-1},\end{cases} \\
& C_{i+1 / 2, j}^{t+\tau}=\left\{\begin{array}{lll}
C_{i, j}^{t+\tau}, & V_{1, i+1 / 2, j} \leq 0, & H_{i, j}>H_{i+1, j}, \\
C_{i+1, j}^{t+\tau}, & V_{1, i+1 / 2, j}>0, & H_{i, j}<H_{i+1, j}, \\
C_{i, j}^{t}, & V_{1, i+1 / 2, j} \leq 0, & H_{i, j}<H_{i+1, j}, \\
C_{i+1, j}^{t}, & V_{1, i+1 / 2, j}>0, & H_{i, j}>H_{i+1, j}
\end{array}\right. \\
& C_{i-1 / 2, j}^{t+\tau}=\left\{\begin{array}{lll}
C_{i-1, j}^{t+\tau}, & V_{1, i-1 / 2, j}>0, & H_{i, j}>H_{i+1, j}, \\
C_{i, j}^{t+\tau}, & V_{1, i+1 / 2, j}<0, & H_{i, j}<H_{i+1, j}, \\
C_{i-1, j}^{t}, & V_{1, i-1 / 2, j}>0, & H_{i, j}<H_{i+1, j}, \\
C_{i, j}^{t}, & V_{1, i+1 / 2, j}<0, & H_{i, j}<H_{i+1, j}
\end{array}\right. \\
& C_{i, j+1 / 2}^{t+\tau}=\left\{\begin{array}{lll}
C_{i, j}^{t+\tau}, & V_{1, i, j+1 / 2} \leq 0, & H_{i, j}>H_{i, j+1}, \\
C_{i, j+1}^{t+\tau}, & V_{1, i, j+1 / 2}>0, & H_{i, j}<H_{i, j+1}, \\
C_{i, j}^{t}, & V_{1, i, j+1 / 2} \leq 0, & H_{i, j}<H_{i, j+1}, \\
C_{i, j+1}^{t}, & V_{1, i, j+1 / 2}>0, & H_{i, j}>H_{i, j+1}
\end{array}\right. \\
& C_{i, j-1 / 2}^{t+\tau}=\left\{\begin{array}{lll}
C_{i, j-1}^{t+\tau}, & V_{1, i, j-1 / 2}<0, & H_{i, j}<H_{i, j-1}, \\
C_{i, j}^{t+\tau}, & V_{1, i, j-1 / 2} \geq 0, & H_{i, j}>H_{i, j-1} \\
C_{i, j-1}^{t}, & V_{1, i, j-1 / 2}<0, & H_{i, j}>H_{i, j-1} \\
C_{i, j}^{t}, & V_{1, i, j-1 / 2} \geq 0, & H_{i, j}<H_{i, j-1}
\end{array} .\right.
\end{aligned}
$$

The computational algorithm is the following. Let the values of the difference functions $U_{i, j}^{t}$, $\Theta_{i, j}^{t}, C_{i, j}^{t}$ and $C_{s, i, j}^{t}$ are determined in the mesh nodes $\left(x_{i}, y_{j}\right)$ at the time point $t$. With these values the flows $V_{1, i \pm 1 / 2, j}, V_{1, i, j \pm 1 / 2}, \omega_{1, i \pm 1 / 2, j}, \omega_{1, i, j \pm 1 / 2}$ and the water flow rate of river through its cross-section $\Gamma_{r}$ are calculated at the boundaries of unit cells from relationships (6), (8). Then, starting with the first cell, we calculate the water amount $\Theta_{i, j}^{t+\tau}$ in zone of incomplete saturation, the thickness $U_{i, j}^{t+\tau}$ of the water layer on the catchment surface and the values of the fluid flows going out from the unit cells. If $U_{i, j}^{t}=0$, then the lag time $t_{0}$ and the retarded 
argument $\tau_{d}$ are additionally computed. The total amount of water crossing the boundary $\Gamma_{r}$ is determined as sum of the corresponding flow-rates with time in the nodes of the shifted grid located at $\Gamma_{r}$. After this the flows $V_{c, i+1 / 2, j}^{t}, V_{c, i, j+1 / 2}^{t}$ and $W_{c, i+1 / 2, j}^{t}, W_{c, i, j+1 / 2}^{t}$ are calculated from (7), (9). At last, the impurity concentration $C_{i, j}^{t+\tau}$ in the rainwater runoff is determined from the system equations (16) and the contaminant concentration $C_{s, i, j}^{t+\tau}$ in fluid moving in the soil - from the formulas (15).

Solution of a problem at the time point $t+\tau$ is finished after the calculation of disbalances to check the computation accuracy, data preparation for computation at next time point and checking the condition of the end of simulation.

\section{RESULTS AND DISCUSSIONS}

The developed numerical model and computational algorithms were implemented in the program package SURFLOW-C to simulate the rainwater runoff along with simultaneous graphic visualization of the computational results. The surface flow and migration of contaminant in the Mesha river basin of Tatarstan Republic are numerically studied with the use of natural data. In particular, the change of the water layer thickness in the intersection of the watershed line and river surface at its lower course has been studied. It is shown that at the initial stage the water level smoothly increases due to the catchment slope in the vicinity of this site of the river. But very soon this level begins sharply rises. After this it gradually stabilizes because of water flow from more outlying sites of the river network and its feeders. The maximal level is reached after stopping of precipitation. In what follows, the thickness of water layer over entire surface catchment gradually decreases.

\section{SUMMARY}

We have developed the finite-difference scheme and the corresponding software to solve the system of nonlinear differential equations with provision for a priori information about the solution behavior. The principal feature of this scheme is the use of the modified grid cells for computation of «the diagonal flows» between the neighbor cells.

In detail the features of the water runoff and the impurity migration in the river basin will be investigated with the use of natural data and presented in our future publications. 


\section{ACKNOWLEDGEMENTS}

The work is performed according to the Russian Government Program of Competitive Growth of Kazan Federal University.

\section{BIBLIOGRAPHY}

[1] V.M. Konyukhov, A.A. Saveliev, M.G. Khramchenkov, A.N. Chekalin, N.E. Galiullina, "Mathematical and Numerical Model of the Rainwater Runoff along the Catchment Surface in the River Basin", Scientific and Technical J. Voprosy Atomnoy Nauki i Tekhniki (VANT), Seriya: Mathematical Modeling of Physical Processes, №. 3, pp. 41-54, 2013 (in Russian).

[2] V.M. Konyukhov, A.A. Saveliev, M.G. Khramchenkov, A.N. Chekalin, "Numerical Modeling of the Water Runoff along the Surface Catchment". In: XIV International Conference. Supercomputing and Mathematical Modeling. (Sarov, Russia, 2012, October, 1 5), Sarov: RFNC-VNIIEF, pp. 374-382, 2013 (in Russian).

[3] A.N. Chekalin, M.G. Khramchenkov, V.M. Konyukhov, I.V. Konyukhov, A.N. Garaeva, "Mathematical Modeling of Rainwater Runoff over Catchment Surface and Mass Transfer Of Contaminant Incoming To Water Stream From Soil”, J. of Fundamental and Applied Sciences, vol. ??, pp. ??-??, 201 ?

[4] S.N. Antontsev, G.P. Epihov, A.A. Kashevarov, "System mathematical modeling of water exchange”, Science, Siberian Branch, Novosibirsk, 1986 (in Russian).

[5] P.S. Eagleson, "Dynamic Hydrology”, McGraw-Hill, New York, 1970.

[6] B. Ambroise, J. Freer, K.J. Beven, "Generalization of the topmodel concepts: topographic indices of hydrological similarity”, Water Resources Research, vol. 32, pp. 2135-2145, 1996.

\section{How to cite this article:}

Chekalin A, Khramchenkov M, Konyukhov V, Konyukhov I, Garaeva A. Numerical model of rainwater runoff over the catchment surface and transport of contaminant incoming to water stream from soil. J. Fundam. Appl. Sci., 2017, 9(2S), 890-902. 